\title{
Commemorating John Dyson
}

\author{
Julian M. Pittard \\ School of Physics and Astronomy, The University of Leeds, Leeds, LS2 9JT, UK \\ email: jmp@ast.leeds.ac.uk
}

John Dyson was born on the $7^{\text {th }}$ January 1941 in Meltham Mills, West Yorkshire, England, and later grew up in Harrogate and Leeds. The proudest moment of John's early life was meeting Freddie Trueman, who became one of the greatest fast bowlers of English cricket. John used a state scholarship to study at Kings College London, after hearing a radio lecture by D. M. McKay. He received a first class BSc Special Honours Degree in Physics in 1962, and began a Ph.D. at the University of Manchester Department of Astronomy after being attracted to astronomy by an article of Zdenek Kopal in the semi-popular journal New Scientist. John soon started work with Franz Kahn, and studied the possibility that the broad emission lines seen from the Orion Nebula were due to flows driven by the photoevaporation of neutral globules embedded in a HII region. John's thesis was entitled "The Age and Dynamics of the Orion Nebula" and he passed his oral examination on $28^{\text {th }}$ February 1966.

John's subsequent career took him to Wisconsin (with a Fulbright Fellowship), Manchester, Munich and Leeds. After studying radio recombination lines at Wisconsin (where John also learnt that the Green Bay Packers were the greatest American football team), in December 1967 John returned to Manchester to take up a lectureship and renewed his interest in dynamics.

One of John's earliest research themes was wind-blown-bubbles (WBBs). Motivated by John Meaburn's discovery of high velocities in HII regions, John led the 1972 Dyson \& de Vries paper "The Dynamical Effects of Stellar Mass Loss on Diffuse Nebulae", a key work in the development of the theory of WBBs. It contains the first similarity solutions describing their dynamics and the first comparison of such models with observations. John applied the basic picture of WBBs to a wide range of sources throughout much of his subsequent career. Though John possessed the ability to attack problems purely analytically, he took interest in observational results, and was very successful at developing models and theories which could be used to make predictions and be tested by data.

John's work in the middle 1980s with Judith Perry on the formation of broad emissionline regions in active galactic nuclei (AGN) due to wind-obstacle interactions was among his most influential. He also wrote papers on AGN emission. His 1985 paper with Pedlar and Unger attributed the optical forbidden line emission in radio Seyfert galaxies to regions shocked by the expansion of the radio emitting components. In related research described in a 1992 paper (Taylor, Dyson \& Axon), John suggested that jets play a role in the formation of some narrow-line regions in Seyferts. In this and other work, John recognized the similarities between wind-driven and jet-driven dynamics. John's other notable work on jet-driven dynamics included the introduction in 1987 of the working surface model of Herbig-Haro objects. He used it to explain observations described in several papers, which he co-authored with observers, on these objects and the jets associated with them.

From the mid-1980s onwards, John contributed greatly to the development of models of flows in multi-phase media. John's thinking on multi-phase WBBs started off by work he did on RCW 58, where it was discovered that the observed correlation of the velocity 
of absorption components with the ionization potential of the ions could not be explained by standard models of wind-blown bubbles. The 1986 Hartquist et al. paper which John co-authored was the first in a series devoted to mass-loaded flows. Such flows are the motions of diffuse material with a large volume filling factor that are influenced by the injection of mass stripped or evaporated from embedded clumps. A series of papers on RCW 58 then followed where models of mass-loaded bubbles were developed and refined. John continued working on developing an understanding of the properties of these largescale flows throughout the 1990s and into the 2000s primarily with Jane Arthur, Robin Williams, and then latterly with myself. I was indeed privileged to have been John's last close collaborator.

John also considered the intermediate-scale structures arising within mass-loaded flows. For instance, in the Dyson et al. (1993) paper he used his physical insight together with simple analytical arguments to elucidate how tails form around the clumps embedded in a subsonic diffuse flow. John was also interested in the changes that occur to a flow when clumps are sufficiently close together that they start to affect the flow in a collective fashion. This is work that we are continuing to investigate at Leeds.

John also did much to bring attention to the potential importance of what have become known as turbulent mixing layers, which he and colleagues referred to as turbulent boundary layers. A 1988 paper by Hartquist \& Dyson contains a number of considerations concerning the mixing in such layers, including ones that are magnetized.

So over a period of more than two decades, John wrote important papers on the general properties of mass-loaded flows, and the intermediate-scale structures and boundary layers within them. He also applied the models to a number of real objects, including Wolf-Rayet nebulae, PNe, SNe, ultracompact HII regions, starburst regions, and the wind of the galactic centre source Sgr A*. By doing so he provided considerable stimulation to several observers. In the last decade of his research he collaborated with Matt Redman and Robin Williams on the nature of magnetohydrodynamic ionization fronts, a problem with the same classic nature as that concerning WBBs which had attracted John's interest 3 decades earlier.

In 1996, Sam Falle, who collaborated with John for more than three decades, managed to convince John to return to West Yorkshire as the Professor of Astronomy in the University of Leeds. John subsequently built up a group which now contains six permanent academic staff, all of whom share star formation as a common interest. Some of the group members also pursue John's other interests in diffuse media. John became an Emeritus Professor in 2006.

John played many roles in the astronomical community. He served on many funding council committees, was on the RAS Council (1975-77), was editor-in-chief of Astrophysics and Space Science for one-and-a-half decades starting in 1993, and was President of IAU Division VI and Commission 34 (2003-6). John also co-authored with David Williams the popular textbook The Physics of the Interstellar Medium.

John was very much a "people person". John, his wife Rita and their children, frequently opened their home to astronomers from many nations, whether short-term guests or people who had just arrived and needed somewhere to stay as they found homes. John was always encouraging and patient, with collaborators and students alike. Most of all, John was always fun to be around, and was known for his rapid and incisive wit.

John passed away peacefully in his sleep at home on the morning of $3^{\text {rd }}$ February 2010.

\section{Acknowledgements}

This commemoration is based on an obituary in the August 2010 issue of Astronomy \& Geophysics written by Tom Hartquist and David Williams. 